\title{
Lower Bounds of Quantum Search for Extreme Point
}

\author{
Yuri Ozhigov *
}

\begin{abstract}
We show that Durr-Hoyer's quantum algorithm of searching for extreme point of integer function can not be sped up for functions chosen randomly. Any other algorithm acting in substantially shorter time $o\left(\sqrt{2^{n}}\right)(n \longrightarrow \infty)$ gives incorrect answer for the functions $\phi$ with the single point of maximum chosen randomly with probability $P_{\text {error }} \longrightarrow 1$. The lower bound as $\Omega\left(\sqrt{2^{n} / b}\right)$ is established for the quantum search for solution of equations $f(x)=1$ where $f$ is a Boolean function with $b$ such solutions chosen at random with asymptotic probability $1(n \longrightarrow \infty)$.
\end{abstract}

\section{Introduction and Background}

In 1996 L.Grover has constructed quantum algorithm which finds the solution of equation $f(x)=1$ in time $O(\sqrt{N})$ where $n$ is the length of word $x$ provided this solution is unique, $N=2^{n}$, (look in $\mathrm{Gr}$ ). His algorithm is the sequential applications of the following steps:

1. $-W R_{0} W$ - diffusion transform.

2. $R_{f}$ - rotation of the phase for solution,

where $W$ is Walsh-Hadamard transform defined by

$$
W=\bigotimes_{j=1}^{n} I_{j}, \quad I_{j}=\left(\begin{array}{cc}
1 / \sqrt{2} & 1 / \sqrt{2} \\
1 / \sqrt{2} & -1 / \sqrt{2}
\end{array}\right),
$$

*Department of mathematics, Moscow state technological University "Stankin", Vadkovsky per. 3a, 101472, Moscow, Russia, e-mail: y@ oz.msk.ru 
$R_{0}(|0\rangle)=-|0\rangle, R_{0}(|e\rangle)=|e\rangle$ for basic states $|e\rangle \neq|0\rangle$, and $R_{f}(|x\rangle)=|x\rangle$ for $f(x)=0$ and $R_{f}(|x\rangle)=-|x\rangle$ for $f(x)=1$.

Soon after this M. Boyer, G. Brassard, P. Hoyer and A. Tapp have shown how arbitrary solution of this equation can be found in time $O(\sqrt{N / t})$ where $t$ is the number of all solutions which is unknown beforehand. They used iterations of Grover's algorithm and measurements which allow to determine the length of the following sequence of iterations ([BBHT]). This algorithm is referred here as G-BBHT. The problem of searching for extreme point of integer function $\phi:\{0,1\}^{n} \longrightarrow\{0,1\}^{n}$ was solved by C. Durr and P. Hoyer in the work [DH. Let an oracle $O_{\phi}$ give a value $\phi(x)$ for every $x \in\{0,1\}^{n}$. The aim is to find a point of maximum of $\phi$.

Their algorithm has the following form. Put $\alpha_{0}=\overline{0}$. Sequentially for $i=0,1, \ldots, T$, do the following. Given $\phi\left(\alpha_{i}\right)$ launch G-BBHT, using oracle $O_{\phi}$ to obtain such $x^{\prime}$ that $\phi\left(x^{\prime}\right) \geq \phi\left(\alpha_{i}\right)$, after that put $\alpha_{i+1}=x^{\prime}$. After that observe the final state. It is shown in [DH] that the point of maximum will be obtained with high probability for $T=O\left(\sqrt{2^{n}}\right)$.

Previously some authors found fast quantum algorithms for other particular problems: P. Shor in the work [Sh], D.Deutsch and R.Jozsa in the work [DJ], D. Simon in the work [Si], and others. Quantum speeding up of such important problem as search has assumed a new significance in the light of the following fact (look at the work [OZ]). No quantum device can predict an evolution of chosen randomly classical system even on one time step. It means that quantum computer can beat classical only with probability zero, and the problem of search turns out to be among such rare cases.

In this work we establish two lower bounds for the quantum search for extreme point. The first result (Theorem 1) says that G-BBHT is optimal in the strong sense: every faster algorithm must fail with probability converging to $1(n \longrightarrow \infty)$. Note that our Theorem 1 may be regarded as a partial amplification of one result from the work [BBHT]. This result is that average time for the quantum search for a solution of $f(x)=1$ for Boolean function $f$ is $d \sqrt{N / b}$ with peculiar constant $d$ in case when there are $b$ such solutions. The second result (Theorem 2) says that Durr-Hoyer's method of searching for an extremum is optimal in the strong sense defined above for the functions with the single point of maximum.

The idea of such lower bounds for quantum algorithms dates back to the work BBBV] of C. Bennett, E. Bernstein, G. Brassard and U. Vazirani. They 
proved that NP-type problem of computing a preimage for length preserving function $f$ can not be solved in time $o(\sqrt{N})$ for $f$ chosen with probability 1 .

In the proofs of Theorems 1 and 2 we use the approach developed in the work [OZ], the idea of Lemma 2 issues from the work [BBBV].

We assume the following basic notions of quantum computing. Each state of quantum computer with $n$ qubits is a point $\chi=\sum_{j} \lambda_{j} e_{j}, \quad\|\chi\|=1$ in $2^{n}$ dimensional Hilbert space with orthonormal basis $\left\{e_{j}\right\}$, where $\lambda_{j}$ are complex numbers called amplitudes. The probability to obtain a basic state $e_{j}$ as a result of observation of the state $\chi$ is $\left|\lambda_{j}\right|^{2}$. A computation has the form $\chi_{0} \longrightarrow \chi_{1} \longrightarrow \ldots \longrightarrow \chi_{t}$ where each passage $\chi_{i} \longrightarrow \chi_{i+1}$ is unitary transform which depends on oracle. A reader can find the more extensive introduction to the quantum computations in the work $\mathrm{Oz}$.

\section{The Effect of Change in Oracles on the Re- sult of Quantum Computations}

To establish the lower bounds for the search of extremum we need some technical notions and propositions concerning the effect of change in oracles on the result of quantum computations which will be considered in this section. We summarize here some facts from the work [Oz] which will be applied in the next section.

We shall denote the basic states by the letter $e$ with indices. Assume that the result of oracle's action on a basic state $e=|\ldots, a, b, \ldots\rangle$ is the state $|\ldots, a, \phi(a)+b, \ldots\rangle$ where $a$ and $b$ are the places for the question and answer respectively, and + means the bitwise addition modulo 2 . This is unitary transformation which is denoted by $\mathrm{Qu}_{\phi}$. Denote this word $a$ by $q(e)$.

A query state $\chi$ is querying the oracle on all the words $q(e)$ with some amplitudes. Put $\mathcal{K}=\{0,1, \ldots, K-1\}$. Let $\chi=\sum_{j \in \mathcal{K}} \lambda_{j} e_{j}$. Given a word $a \in\{0,1\}^{n}$ for a query state $\chi$ we define:

$$
\delta_{a}(\chi)=\sum_{j: q\left(e_{j}\right)=a}\left|\lambda_{j}\right|^{2} .
$$

It is the probability that a state $\chi$ is querying the oracle on the word $a$. In particular, $\sum_{a \in\{0,1\}^{n}} \delta_{a}(\chi)=1$. 
Each query state $\chi$ induces the metric on the set of all oracles if for functions $f, g$ of the form $\{0,1\}^{n} \longrightarrow\{0,1\}^{n}$ we define a distance between them by

$$
\mathrm{d}_{\chi}(f, g)=\left(\sum_{a: f(a) \neq g(a)} \delta_{a}(\chi)\right)^{1 / 2} .
$$

Lemma 1 Let $\mathrm{Qu}_{f}, \mathrm{Qu}_{g}$ be query transforms on quantum part of $Q C$ corresponding to functions $f, g ; \chi$ be a query state. Then

$$
\left\|\mathrm{Qu}_{f}(\chi)-\mathrm{Qu}_{g}(\chi)\right\| \leq 2 \mathrm{~d}_{\chi}(f, g) .
$$

\section{Proof}

Put $\mathcal{L}=\left\{j \in \mathcal{K} \mid f\left(q\left(e_{j}\right)\right) \neq g\left(q\left(e_{j}\right)\right)\right\}$. We have: $\left\|\mathrm{Qu}_{f}(\chi)-\mathrm{Qu}_{g}(\chi)\right\| \leq$ $2\left(\sum_{j \in \mathcal{L}}\left(\left|\lambda_{j}\right|\right)^{2}\right)^{1 / 2} \leq 2 \mathrm{~d}_{\chi}(f, g)$. Lemma is proved.

A quantum computation has the form

$$
\chi_{0} \longrightarrow \chi_{1} \longrightarrow \ldots \longrightarrow \chi_{t},
$$

where each step $\chi_{i} \longrightarrow \chi_{i+1}$ is the superposition of the query unitary transform and the following unitary transform $U_{i}$ which depends only on $i: \chi_{i} \stackrel{\mathrm{Qu}_{f}}{\longrightarrow}$ $\chi_{i}^{\prime} \stackrel{U_{i}}{\longrightarrow} \chi_{i+1}$. We shall denote $U_{i}\left(\mathrm{Qu}_{f}(\chi)\right)$ by $V_{i, f}(\chi)$, then $\chi_{i+1}=V_{i, f}\left(\chi_{i}\right), i=$ $0,1, \ldots, t-1$. Here $t$ is the number of query transforms (or evaluations of the function $f$ ) in the computation at hand. We say that the number $t$ is the time complexity of this computation.

Put $\mathrm{d}_{a}(\chi)=\sqrt{\delta_{a}(\chi)}$.

Lemma 2 If $\chi_{0} \longrightarrow \chi_{1} \longrightarrow \ldots \longrightarrow \chi_{t}$ is a computation with oracle for $f$, a function $g$ differs from $f$ only on one word $a \in\{0,1\}^{n}$ and $\chi_{0} \longrightarrow \chi_{1}^{\prime} \longrightarrow$ $\ldots \longrightarrow \chi_{t}^{\prime}$ is a computation on the same $Q C$ with a new oracle for $g$, then

$$
\left\|\chi_{t}-\chi_{t}^{\prime}\right\| \leq 2 \sum_{i=0}^{t-1} \mathrm{~d}_{a}\left(\chi_{i}\right)
$$




\section{Proof}

Induction on $t$. Basis is evident. Step. In view of that $V_{t-1, g}$ is unitary, Lemma 1 and inductive hypothesis, we have

$$
\begin{aligned}
& \left\|\chi_{t}-\chi_{t}^{\prime}\right\|=\left\|V_{t-1, f}\left(\chi_{t-1}\right)-V_{t-1, g}\left(\chi_{t-1}^{\prime}\right)\right\| \leq \\
& \left\|V_{t-1, f}\left(\chi_{t-1}\right)-V_{t-1, g}\left(\chi_{t-1}\right)\right\|+\left\|V_{t-1, g}\left(\chi_{t-1}\right)-V_{t-1, g}\left(\chi_{t-1}^{\prime}\right)\right\| \leq \\
& 2 \mathrm{~d}_{a}\left(\chi_{t-1}\right)+\left\|\chi_{t-1}-\chi_{t-1}^{\prime}\right\|=2 \mathrm{~d}_{a}\left(\chi_{t-1}\right)+2 \sum_{i=0}^{t-2} \mathrm{~d}_{a}\left(\chi_{i}\right)=2 \sum_{i=0}^{t-1} \mathrm{~d}_{a}\left(\chi_{i}\right) .
\end{aligned}
$$

Lemma is proved.

In what follows we assume that all computations are performed with fixed probability of error $p_{\text {err }}$. This means that if $B$ is the set of numbers of target states then the probability $\sum_{j \in B}\left|\lambda_{j}\right|^{2}$ to obtain one of such states as a result of observations of final state $\chi_{t}=\sum_{j} \lambda_{j} e_{j}$ is not less than $1-p_{e r r}$.

\section{Strong Lower Bound for the Time Com- plexity of the Quantum Search}

At first take up the problem of search for the extreme point of Boolean functions. Given an oracle for function $\phi:\{0,1\}^{n} \longrightarrow\{0,1\}$ from some fairly wide set $S$, what is the lower bound for the time complexity of quantum search for its extreme point? We shall require that our algorithms give the correct answer not on all functions $\phi$ but only on the functions from some set $G \subseteq S$. Suppose that we fixe two constants:

1) the maximal admitted probability of error $\epsilon>0$ (for the computations with oracles for $\phi \in G$ ), and

2) the probability of applicability of the algorithm: $\operatorname{card}(G) / \operatorname{card}(S)$ such that this ratio must be at most $p$ for some $p: 0<p \leq 1$.

If $S$ is the set of all Boolean functions the best possible lower bound in quantum case as well as in classical is $O(1)$. This is because the simple classical algorithm verifying $\phi(0), \phi(1), \ldots, \phi(k)$ gives the correct answer for the functions chosen with probability $p=1-2^{-k}$.

Let $S=S_{b}$ be the set of all Boolean functions with exactly $b$ points $x$ such that $\phi(x)=1$. Let further $n, t(n), b(n)$ vary such that $t=o(\sqrt{N / b}), n \longrightarrow$ $\infty, N=2^{n}$. A quantum algorithm with the time complexity $t(n)$ thus is 
substantially faster than G-BBHT. We shall prove that if we apply such algorithm to the search for extremum of $\phi$ it must make a mistake for a bulk of $\phi$.

Theorem 1 Let $t(n)=o(\sqrt{N / b(n)}), n \longrightarrow \infty$, and some quantum computer with oracle for $\phi$ with the time complexity $t(n)$ searchs for a solution of $\phi(x)=1$ with fixed upper bound $\epsilon$ for the probability of error $(0<\epsilon<1)$. Let $p(n)$ be the probability of that this algorithm gives the correct answer for the oracle $\phi$ chosen randomly from $S_{b}$. Then $p(n) \longrightarrow 0(n \longrightarrow \infty)$.

\section{Proof}

We shall apply the idea of proof of Theorem 2 from the work $\mathrm{Oz}$ with some modifications. Fix $n$ and put $\phi_{0}(x)=0$. Let $X_{0} \longrightarrow X_{1} \longrightarrow \ldots \longrightarrow$ $X_{t}$ be the computation on quantum machine at hand. Define the matrix $a_{i j}=\delta_{j}\left(X_{i}\right), i=1,2, \ldots, t ; j=1,2, \ldots, N$, where $N=2^{n}$. Then we have $\sum_{i j} a_{i j} \leq t$ because $\forall i \sum_{j} a_{i j} \leq 1$.

Let $T_{j}$ be the set of all such integers $\tau$ that $\sum_{i} a_{i \tau} \leq(j+1) t / N$; assume $T_{0}=\emptyset$. Let $\hat{b}_{j}$ denote the cardinality of the set $L_{j}=T_{j} \backslash T_{j-1}$. Then $\sum_{j} \frac{\hat{b}_{j}(j+1) t}{N} \leq t$.

Choose randomly $b$ different integers from $1,2, \ldots, N$ denote this set by $D$ and let $b_{j}$ be the number of such integers among them which belongs to the set $L_{j}$. Then $b_{j}$ is a random variable with the expectation $\mathrm{E} b_{j}=b \hat{b}_{j} / N$. Now change the values of $\phi_{0}$ on $D$ to 1 . We obtain a new function $\phi_{1}$ and correspondingly the new computation $X_{0}^{\prime}=X_{0} \longrightarrow X_{1}^{\prime} \longrightarrow \ldots \longrightarrow X_{t}^{\prime}$ with oracle for $\phi_{1}$. The norm of difference between the final states $\xi=\left\|X_{t}-X_{t}^{\prime}\right\|$ will be thus a real random variable. Estimate its expectation.

Lemma 3 For every $\varepsilon>0 P(\xi>\varepsilon) \longrightarrow 0$ if $n \longrightarrow \infty$.

Proof

We need the following inequality for every random variable: $\mathrm{E} \eta^{2} \geq \mathrm{E}^{2} \eta$.

Let $i$ takes all values $1,2, \ldots, N ; j$ takes all natural values. We have:

$$
\begin{aligned}
& \mathrm{E} \xi=2 \mathrm{E} \sum_{i} \sqrt{\sum_{\tau \in D} a_{i \tau}} \leq 2 \mathrm{E} \sqrt{t \sum_{j} b_{j}(j+1) t / N}=\frac{t \sqrt{b}}{\sqrt{N}} 2 \mathrm{E} \sqrt{\sum_{j} b_{j}(j+1) / b} \leq \\
& o(1) \sqrt{\mathrm{E} \sum_{j} b_{j}(j+1) / b} \leq o(1) \sqrt{\frac{1}{b} \sum_{j} \frac{b \hat{b}_{j}(j+1)}{N}}=o(1)(n \longrightarrow \infty) .
\end{aligned}
$$


Now applying Chebishev inequality $P(\xi \geq \varepsilon) \leq \mathrm{E} \xi / \varepsilon$ we conclude that if $\varepsilon$ is fixed then $P(\xi \geq \varepsilon)$ may be done arbitrarily small for sufficiently large $n$. Lemma 3 is proved.

Turn to the proof of Theorem 1. Suppose that our computer gives the correct answer on all functions from $G$ with probability $p_{\text {err }}$ of error. Without loss of generality we may assume $p_{e r r}=0.0016, N>1000$. Choose a Boolean function $f \in G$ which takes the value 1 in $b$ points. Let the final state of computation on our computer with oracle $f$ has the form $X_{t}=\sum_{j} \lambda_{j} e_{j}$. Let $B=\left\{j \mid f\left(e_{j}\right)=1\right\}, \quad \varepsilon_{0}=\sum_{j \notin B}\left|\lambda_{j}\right|^{2}$. We have

$$
\varepsilon_{0} \leq p_{e r r}
$$

because the final observation of $X_{t}$ must give the result $e_{j}, j \in B$ with probability of error $p_{\text {err }}$. Fix such $f$ and put $c_{j}=j / N, j=0,1, \ldots ; \quad L_{j}=$ $\left\{\left.j \in B\left|c_{j} \leq\right| \lambda_{j}\right|^{2}<c_{j+1}\right\}, \zeta_{0}=\sum_{j} \hat{l}_{j} c_{j}$ where $\hat{l}_{j}=\operatorname{card}\left(L_{j}\right)$. We have

$$
\left|1-\zeta_{0}\right| \leq \varepsilon_{0}+\frac{b}{N}<2 p_{e r r} \quad(N \longrightarrow \infty)
$$

Now choose the second function $f^{\prime} \in S_{b}$ randomly. Let $B^{\prime}=\left\{j \mid f^{\prime}\left(e_{j}\right)=\right.$ $1\}$. Define a random variables $l_{j}$ depending on $f^{\prime}$ :

$$
l_{j}=\operatorname{card}\left\{j \mid j \in L_{j} \cap B^{\prime}\right\} .
$$

We have $\mathrm{E} l_{j}=b \hat{l}_{j} / N$ because the probability of the choice of $f^{\prime}$ is uniformly distributed over all $S_{b}$. At last define $\zeta=\sum_{j} l_{j} c_{j}$. This is also a random variable depending on $f^{\prime}$. Its expectation is

$$
\mathrm{E} \zeta=\sum_{j} c_{j} \mathrm{E} l_{j}=\sum_{j} \frac{c_{j} b \hat{l}_{j}}{N}=O(1) b / N=o(1) \quad(N \longrightarrow \infty)
$$

in view of (2). Then Chebishev inequality $P(\zeta \geq 0.9) \leq \frac{10}{9} \mathrm{E} \zeta$ gives

$$
P(\zeta \geq 0.9) \longrightarrow 0(N \longrightarrow \infty)
$$

Now suppose that $\operatorname{card}(G) / \operatorname{card}\left(S_{b}\right)=\epsilon_{0}=$ const. 
Let $X_{t}^{\prime}=\sum_{j} \lambda_{j}^{\prime} e_{j}$ be the final state of the computation with oracle for a chosen function $f^{\prime}$. If $f^{\prime} \in G$ (e.g. with probability $\epsilon_{0}$ ) then we have

$$
0 \leq \sum_{j \notin B^{\prime}}\left|\lambda_{j}^{\prime}\right|^{2} \leq p_{e r r}
$$

Applying Lemma 3 to the random variable $\xi$ depending on the choice of $f^{\prime}$ we have that with probability 1

$$
\xi^{2} \longrightarrow 0(N \longrightarrow \infty)
$$

We have

$$
\begin{gathered}
\xi^{2}=\left\|X_{t}-X_{t}^{\prime}\right\|^{2}=\sum_{j \in B \backslash B^{\prime}}\left|\lambda_{j}-\lambda_{j}^{\prime}\right|^{2}+\sum_{j \in B^{\prime} \backslash B}\left|\lambda_{j}-\lambda_{j}^{\prime}\right|^{2}+ \\
\sum_{j \notin B \cup B^{\prime}}\left|\lambda_{j}-\lambda_{j}^{\prime}\right|^{2}+\sum_{j \in B \cap B^{\prime}}\left|\lambda_{j}-\lambda_{j}^{\prime}\right|^{2} . \\
\text { Put } \sum_{j \in B^{\prime} \backslash B}\left|\lambda_{j}^{\prime}\right|^{2}=q^{\prime}, \sum_{j \notin B \cup B^{\prime}}\left|\lambda_{j}^{\prime}\right|^{2}=z^{\prime}, \sum_{j \in B^{\prime} \backslash B}\left|\lambda_{j}\right|^{2}=q, \sum_{j \notin B \cup B^{\prime}}\left|\lambda_{j}\right|^{2}=z, \\
\sum_{j \in B \cap B^{\prime}}\left|\lambda_{j}\right|^{2}=r, \sum_{j \in B \cap B^{\prime}}\left|\lambda_{j}^{\prime}\right|^{2}=r^{\prime} .
\end{gathered}
$$

Then in view of (1) $q \leq \varepsilon_{0} \leq p_{\text {err }}$ and $z \leq p_{\text {err }}$. We shall use inequality $\|a-b\| \geq|\|a\|-\|b\||$ for two vectors $a, b$ in Hilbert space. Using this inequality we conclude that the second item in (6) is not less than $\delta=\left|\sqrt{q^{\prime}}-\sqrt{q}\right|^{2}$. The third item is not less than $\left|\sqrt{z^{\prime}}-\sqrt{z}\right|^{2}$. Let $N$ be sufficiently large, such that

$$
\xi^{2}<p_{\text {err }} .
$$

Such $N$ exists by (5). Then we have $q^{\prime}<4 p_{\text {err }}$. Really, in opposite case: $q^{\prime} \geq 4 p_{\text {err }}$ in view of (6) we would have $p_{\text {err }}>\delta \geq\left(\sqrt{q^{\prime}}-\sqrt{p_{\text {err }}}\right)^{2} \geq p_{\text {err }}$ which gives contradiction. Similarly, $z^{\prime}<4 p_{\text {err }}$. Hence asymptotically when $N \longrightarrow \infty$ with probability 1: $\sum_{j \notin B}\left|\lambda_{j}^{\prime}\right|^{2}=q^{\prime}+z^{\prime}<8 p_{\text {err }}$. Therefore with this probability $\sum_{j \in B}\left|\lambda_{j}^{\prime}\right|^{2}>1-8 p_{e r r}$. Taking into account (4) we obtain that with probability $\epsilon_{0}$

$$
r^{\prime}>1-9 p_{e r r}
$$

From the definition of $L_{j}$ it follows that

$$
|\zeta-r|<\frac{1}{N}
$$


On the other hand (6) and (7) give $\left|r-r^{\prime}\right|=\left|\sqrt{r}-\sqrt{r^{\prime}}\right|\left(\sqrt{r}+\sqrt{r^{\prime}}\right)$ $<2 \sqrt{s} \leq 2 \sqrt{p_{\text {err }}}$ where $s$ is the fourth item in the sum (6). Now (9) gives $\left|\zeta-r^{\prime}\right|<\frac{1}{N}+2 \sqrt{p_{e r r}}$, and by (8) $\zeta>1-9 p_{\text {err }}-2 \sqrt{p_{\text {err }}}-\frac{1}{N}>0.903$ with probability $\epsilon_{0}$, which contradicts to $(3)$. Theorem 1 is proved.

\section{Lower Bound of the Quantum Search for the Single Extreme Point}

Now we are ready to give the lower bound for the problem of search for extreme point of the integer function. We assume that $\phi$ is arbitrary integer function with the single point of maximum and there are the probability measure distributed uniformly on the set of all such functions, so that each $\phi$ can be chosen with the same probability. The set of all such functions is denoted by $C$.

Theorem 2 If some quantum algorithm with the time complexity o $(\sqrt{N})$ finds a point of maximum for the functions from $C$ with probability of applicability $p(n)$ then $p(n) \longrightarrow 0(n \longrightarrow \infty)$.

\section{Proof}

Let $C_{l}$ be the set of such functions from $C$ whose maximum is $N-l$. It is sufficient to prove the Theorem for each $C_{l}$ separately, $l=1,2, \ldots, N$. The cases of all $C_{l}$ are analogous, let, for example, $l=1$.

We shall use Theorem 1. Fix some quantum algorithm. Let $f$ be such integer function that does not take the value $N-1$. The set of all such functions is denoted by $H$. If we redefine such $f$ on a single point and obtain a function $\phi \in C_{1}$ we say that this function $\phi$ is generated by $f$. Denote the set of all $N$ such functions by $[f]$. From the proof of Theorem 1 it follows that for every $\epsilon>0$ there exists such $n_{0}$ that for each $n>n_{0}$ and $f \in H$ the probability of that our quantum algorithm finds a point of maximum for randomly chosen function in $[f]$ will be less than $\epsilon$.

Let $M$ be the number of all different sets $[f], K$ be the cardinality of $S_{1}$. Then each $\phi \in C_{1}$ belongs to exactly $N-1$ sets of the form $[f]$. Now count all functions $\phi \in C_{1}$ for which our algorithm does not find a point of maximum by two different ways. At first count all such $\phi$ in each $[f]$ and add all results. We obtain at least $M N(1-\epsilon)$ and here each such $\phi$ is 
counted exactly $N-1$ times. But $M N=K(N-1)$, therefore the number of such $\phi$ is $K(1-\epsilon)$. Here $\epsilon$ can be made arbitrarily small and we obtain $p(n)=\epsilon \longrightarrow 0 \quad(n \longrightarrow \infty)$. Theorem 2 is proved.

\section{Acknowledgements}

I would like to thank Lov Grover whose questions stimulated me, and who informed me about the work [DH]. I am grateful to academician Victor Maslov for his attention and support, to professor Oleg Chrustalev for discussions on quantum computers, and especially to the principal of "Stankin" Yuri Solomentsev for the financial support of my work.

\section{References}

[BBBV] Bennett, C. H., Bernstein, E., Brassard, G., Vazirani, U. 1997 Strengths and Weaknesses of Quantum Computing. SIAM Journal on Computing 26(5), 1510-1523, (lanl e-print quant-ph/9701001).

[BBHT] Boyer, M., Brassard, G., Hoyer, P., Tapp, A. 1996 Tight bounds on quantum searching. In Fourth Workshop on Physics and Computation (ed. T. Toffoli \& M. Biaford \& J. Leao), pp. 36-43. New England Complex Systems Institute.

[DH] Durr, C., Hoyer, P., A Quantum Algorithm for Finding the Minimum (lanl e-print quant-ph/9607014).

[DJ] Deutsch, D., Jozsa, R., 1992 Rapid solution of problems by quantum computation. Proc. Roy. Soc. Lond. A 439, 553-558.

[Gr] Grover, L. K. 1996 A fast quantum mechanical algorithm for database search. Proceedings, STOC 1996, 212-219. Philadelphia PA USA.

[Oz] Ozhigov, Y. 1998 Quantum Computers Speed Up Classical with Probability Zero, To be published in "Chaos, Solitons and Fractals", issue on Quantum Computing, (lanl e-print quant-ph/9803064).

[Sh] Shor, P.W., 1994 Polynomial-Time Algorithms for Prime Factorization and Discrete Logarithms on Quantum Computer In Proceedings of the 
35th Annual Symposium on Foundations of Computer Science, Santa Fe, NM, Nov. 20-22, 1994, pp 124-134. IEEE Computer Society Press.

[Si] Simon, D., 1994 On the power of quantum computations. In Proceedings of the 35th Annual Symposium on the Foundations of Computer Science, p. 116. IEEE Computer Society Press, Los Alamos, CA. 